\title{
Quantitative STEM of Catalyst Nanoparticles using ADF Imaging with Simultaneous EDS and EELS Spectroscopy.
}

\author{
Aakash M Varambhia ${ }^{1}$, Lewys Jones ${ }^{1}$, Annick De Backer ${ }^{2}$, Sandra Van Aert ${ }^{2}$, Dogan Ozkaya ${ }^{3}$, \\ Sergio Lozano-Perez ${ }^{1}$, Peter D Nellist ${ }^{1}$ \\ ${ }^{1}$ Department of Materials, Oxford University, Parks Road, Oxford OX1 3PH, UK \\ ${ }^{2}$ EMAT, University of Antwerp, Groenenborgerlaan 171, B-2020 Antwerp, Belgium \\ ${ }^{3}$ Johnson Matthey Technology Centre, Blounts Court Road, Sonning Common, Reading RG4 9NH, \\ UK
}

Some of the key properties that affect the catalytic performance of nanoparticle ensembles are particle size, shape, surface-strain and composition. These parameters need to be measured from an ensemble of nanoparticles to obtain useful parameters to compare to catalytic activity. Using Scanning Transmission Electron Microscopy (STEM) it is possible to measure these parameters simultaneously from a nanoparticle. The Annular Dark Field (ADF) image can be used to obtain atomic positions [1,2], whereas Energy Dispersive X-ray (EDS) and Electron Energy Loss Spectroscopy (EELS) can be used to obtain high resolution compositional information. With careful instrument calibrations all three of these signals can be converted into quantitative scattering crosssections $[3,4]$ to count the number of atoms along an atomic column of a nanoparticle.

Such atomic column counts can then be modelled into three-dimensional structures (Figure 1). We present results utilising experimental models of pure Pt nanoparticles as inputs for density functional theory (DFT) simulations for calculating oxygen binding energies. The experimental models were obtained using recent advances in detector calibration methods developed at Oxford University giving an $\mathrm{ADF}$ atom counting accuracy of $0.1 \%$.

Building on previous literature [5], a best practice methodology to determine the scattering crosssections for EDS and EELS will also be presented. Using these cross-sections some initial results combining EDS, EELS and ADF cross-sections simultaneously for Pt-Co binary alloy nanoparticles will be discussed. These results were obtained using simultaneous multi-frame acquisitions, where the individual frames were compensated for scan-distortion [6]. Post-acquisition inspection allows for only those frames with minimal damage from the electron beam to be included in the quantification, further improving experimental precision and reliability. (Figure 2). Finally, some high-throughput analysis techniques such as particle sizing (figure 3) will be presented, with the aim of linking individual particle properties to whole ensemble properties.

References:

[1] L. Jones et al, Nanolett, 2014, 14, 6336.

[2] A.M. Varambhia et al, Particle \& Particle Systems Characterization, 2016, 33, 438-444.

[3] H. E et al, Ultramicroscopy, 2013, 133, 109.

[4] K. E. MacArthur et al, Microsc. Microanal, 2016, 22, 71.

[5] A. J. Craven et al, Ultramicroscopy, 2016, 170, 113.

[6] L. Jones et al, Advanced Structural and Chemical Imaging, 2015, 1:8 


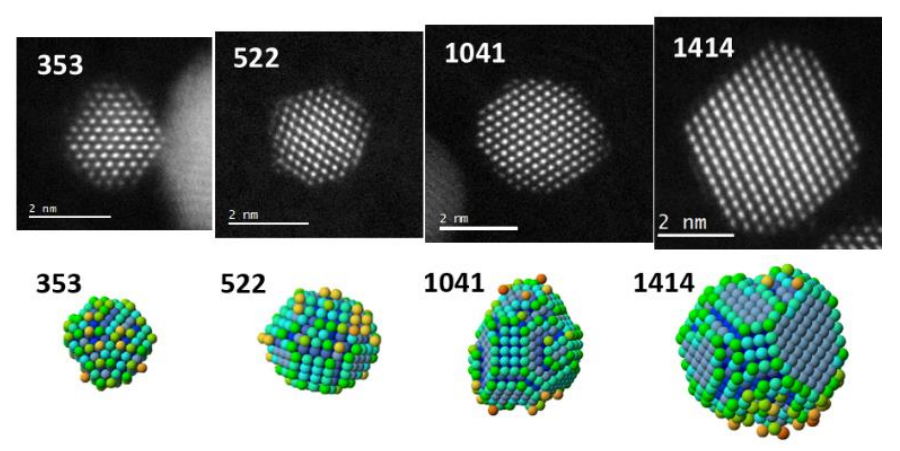

Figure 1. Atom counted Pt nanoparticles with their corresponding models created using the methodology similar to ref [1].

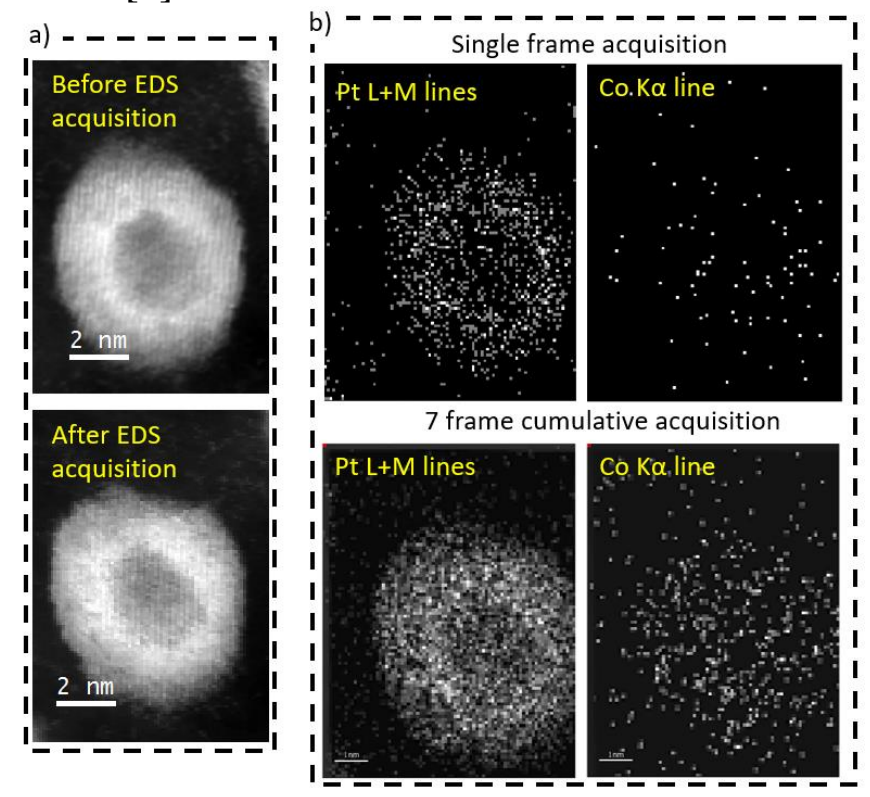

Figure 2. a) ADF images of a Pt-Co nanoparticle before and after multi-frame acquisition, b) Raw EDS signal from single frame and cumulative frame acquisition.

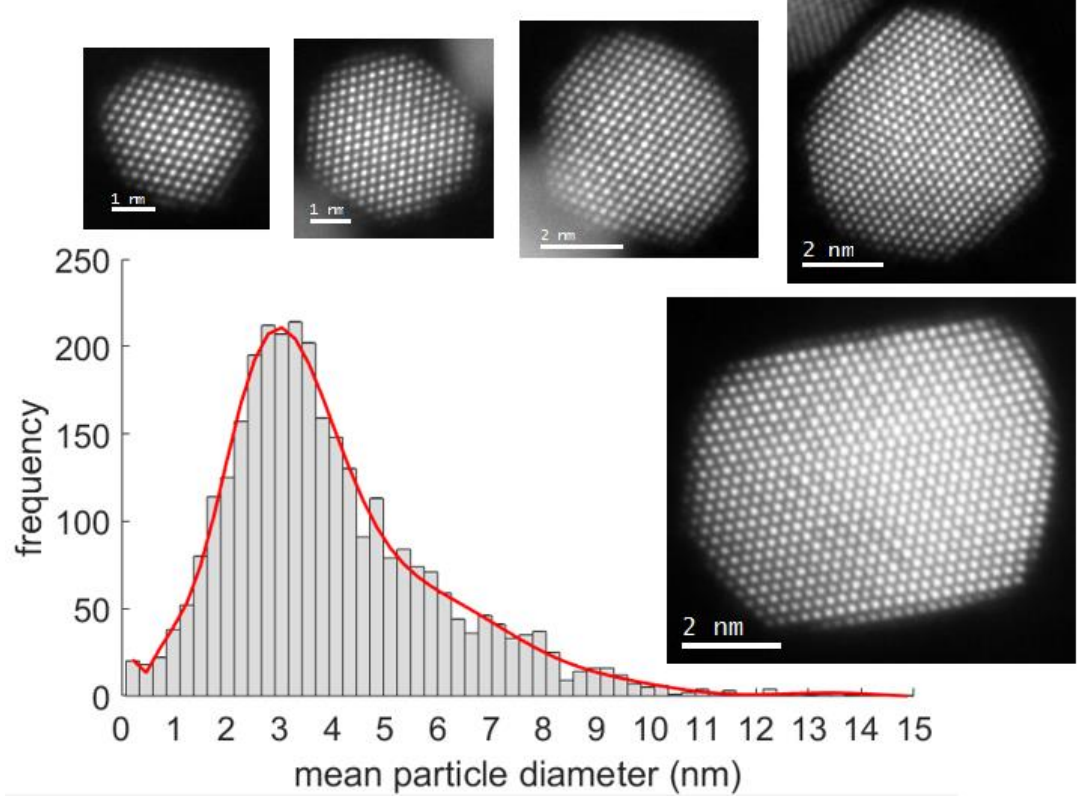

Figure 3. Particle sizing histogram of Pt3Co nanoparticles with size selected atomic resolution images 